\title{
A Study to decrease the incidence of PPH in our hospital setting
}

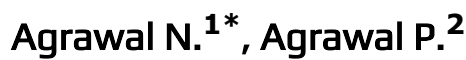

DOI: https://doi.org/10.17511/joog.2021.i04.01

\footnotetext{
1* Neelima Agrawal, Consultant, Department of Obstetrics and Gynaecology, Agrawal Hospital, Bhopal, Madhya Pradesh, India.

2 P. N. Agrawal, Consultant Respiratory and Critical care, Agrawal Hospital, Bhopal, Madhya Pradesh, India.
}

Objective: This study aimed to measure incidence, identify risk factors (pre-existing or acquired) for PPH and to prevent progression to severe PPH. Methods: This is a prospective observational study. The causes are likely to be multifactorial with shifting demography and health status widely cited, e.g. age, obesity, comorbidity, multiple pregnancy and ethnicity. Results: Prepregnancy factors for PPH include age, ethnicity, BMI, previous PPH and assisted conception Conclusion: Prophylactic uterotonic agents in high-risk patients, use of intramuscular or intravenous oxytocin, timely application of B -lynch suture, adequate and timely arrangement of blood and blood factors can help prevent PPH.

Keywords: Postpartum haemorrhage (PPH), Maternal mortality, Uterotonic agents, B -lynch suture

Corresponding Author

Neelima Agrawal, Consultant, Department of Obstetrics and Gynaecology, Agrawal Hospital, Bhopal, Madhya Pradesh, India.

Email: sumit_bhargava782000@yahoo.com
How to Cite this Article

To Browse

Neelima Agrawal, P N Agrawal, A Study to decrease the incidence of $\mathrm{PPH}$ in our hospital setting. Obs Gyne Review J Obstet Gynecol. 2021;7(4):35-41. Available From

https://obstetrics.medresearch.in/index.php/joog/art icle/view/141

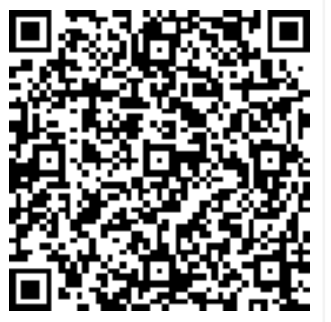

Manuscript Received 2021-04-15

Conflict of Interest No

(c) 2021 by $\mathrm{Ne}$
Review Round 1 2021-04-30

Funding Nil
Review Round 2 2021-05-16

Ethical Approval Yes

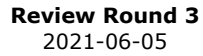

Plagiarism X-checker $9 \%$
Accepted 2021-06-30

Note 


\section{Introduction}

Postpartum haemorrhage (PPH) is the leading cause of maternal mortality worldwide. Postpartum haemorrhage (PPH), defined as blood loss $\geq 500 \mathrm{ml}$, is a major cause of maternal mortality and morbidity worldwide. For every death, 20 women live with the consequences of associated morbidities, with the greatest burden in low-income countries. PPH is a common emergency, and readily treatable when appropriate resources are available. [1]. Severe PPH (variously defined from $1000 \mathrm{ml}$ upwards) has been used as a measure of severe morbidity. Despite surgical, medical and training innovations, PPH rates remain high in several countries. The causes are likely to be multifactorial with shifting demography and health status widely cited, e.g. age, obesity, comorbidity, multiple pregnancy and ethnicity.[2-4].

A better understanding of the factors associated with PPH maternal mortality is critical for preventing the risk of hospital-based maternal death. The purpose of this study was thus to assess which factors contribute to maternal death occurring during PPH. The factors were as follows: women's characteristics, aspects of pregnancy and delivery; components of PPH management; and organizational characteristics of the referral hospitals. This prospective observational study aimed to: (i) measure PPH incidence; (iii) identify chronologically ordered risk factors (pre-existing or acquired) for PPH and progression to severe PPH. Identifying risk pathways is important as predisposing risk can underlie factors that appear, accumulate and dominate later events, including subsequent pregnancies. Judicious use of uterotonics, aggressive approach, application of B lynch suture and timely arrangement of blood and blood products is essential. [3-8].

\section{Materials and Methods}

- Duration and type of study: This is a prospective observational study. The causes are likely to be multifactorial with shifting demography and health status widely cited, e.g. age, obesity, comorbidity, multiple pregnancy ethnicity etc.

- Setting: Hospital setting

- Sampling methods: Patients shifted from OT to ICU and for how long. The patients with severe blood loss needed blood transfusions and other blood products like FFP, platelets etc.
We also divided the groups based on normal or caesarean deliveries, the procedure done and medicines given and the outcome was noted.

- Inclusion criteria: All patients fulfilling the PPH criteria and required some intervention were included

- Exclusion criteria: Deliveries and LSCS which had blood loss of less than $500 \mathrm{ml}$ were excluded

- Data collection procedure: All delivery and LSCS patients were studied and those fulfilling the PPH criteria were included in the study.

- Any scoring system: PPH was quantified into mild $<500 \mathrm{ml}$, moderate $500 \mathrm{ml}$ to $1000 \mathrm{ml}$ and severe $>1000 \mathrm{ml}$.

- Surgical procedure if any: Judicious use of uterotonics, aggressive approach, application of $B$ lynch suture and timely arrangement of blood and blood products is essential.

- Ethical consideration \& permission: Not required as it was an observational study

- Statistical Analysis: Data was compiled using MS excel 2007 and analysis was done with the help of Epi-Info 7 software. Frequency and percentage were calculated \& a statistical test (Chi-Square) was applied wherever applicable; $p<0.05$ was taken as statistically significant.

\section{Results}

Ours is a multispeciality hospital with most of the patients being respiratory. Obstetrics and Gynaecology is an integral part of the hospital as one of the directors is a gynaecologist.The ICU is one of the best in the city and therefore all types of high risk obstetrics is being taken here. The above chart shows number of patients admitted and the division between total patients and obs. \& gynae. patients to give an idea of propotion of both the streams.

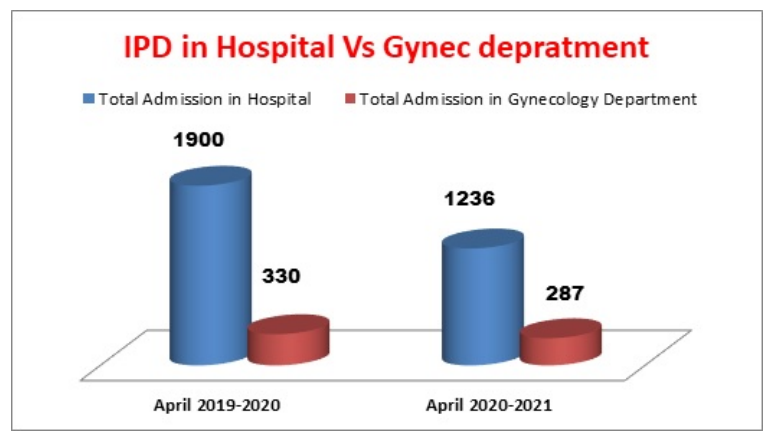




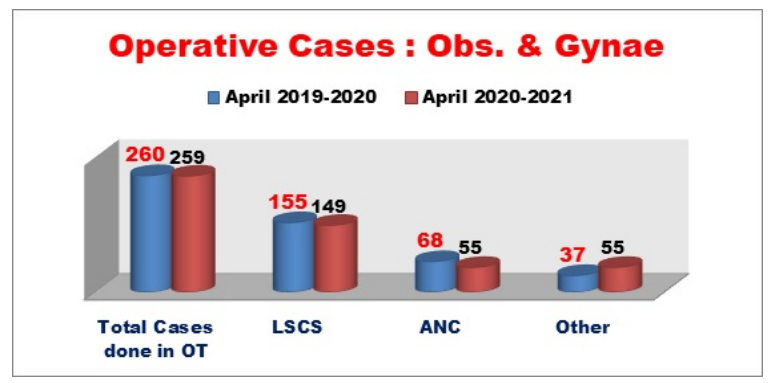

After many infrastructural changes and increase in beds, now the gynaecology department is strengthened and number of cases likely to increase.

The above chart shows the trend in both the years as far as obs \&gynae cases are concerned. It is almost similar and shows the no. of LSCS and normal deliveries.

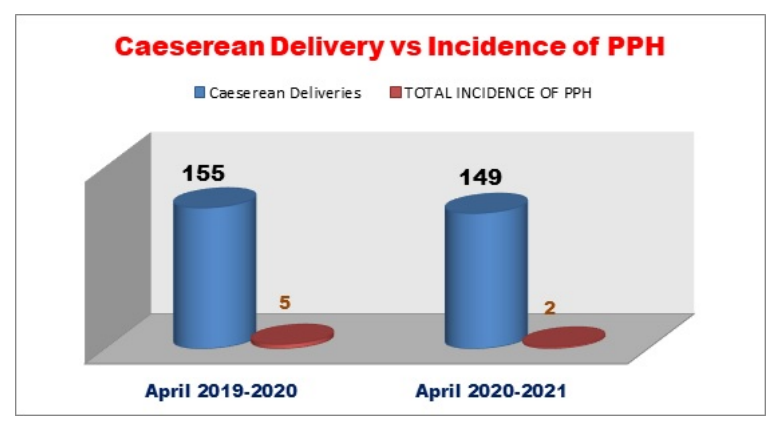

This chart shows the total no. of deliveries and incidence of PPH in two year period

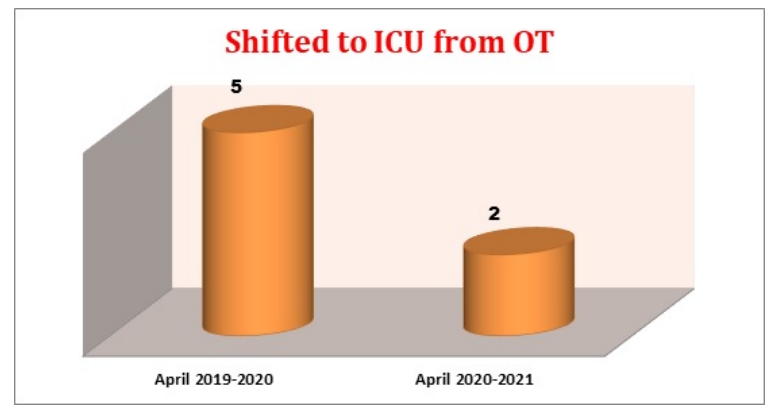

The ICU is one of the best in the city and therefore all types of high-risk obstetrics is being taken here. 5 patients were shifted last year whereas this year it was only 2 patients, showing improvement in care.

Due care preoperatively with timely management necessitated blood transfusion only in one patient this year whereas blood transfusion was required in 4 patients last year.

The three highest-ranked uterotonic agents for prevention of $\mathrm{PPH} \geq 500 \mathrm{~mL}$ were ergometrine plus oxytocin combination, misoprostol plus oxytocin
Combination and carbetocin.

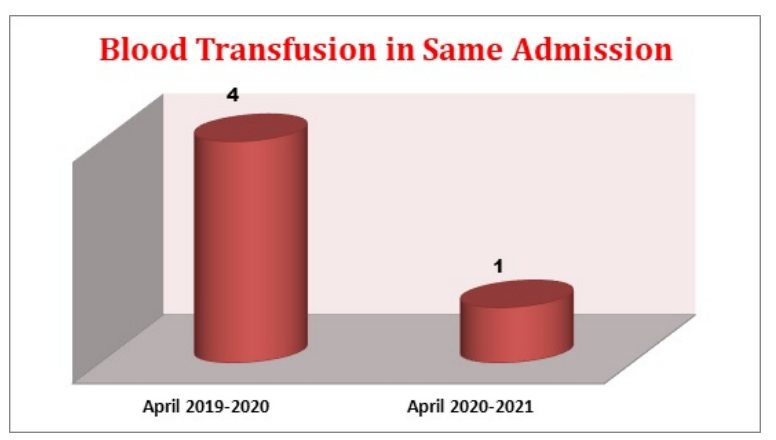

There is evidence that ergometrine plus oxytocin (RR $0.70,95 \%$ CI 0.59 to 0.84 , moderate certainty), carbetocin (RR $0.72,95 \%$ CI 0.56 to 0.93 , moderate certainty) and misoprostol plus oxytocin (RR $0.70,95 \%$ CI 0.58 to 0.86 , low certainty) may reduce $\mathrm{PPH} \geq 500 \mathrm{~mL}$ compared with oxytocin. A better understanding of the factors associated with PPH maternal mortality is critical for preventing the risk of hospital-based maternal death. The purpose of this study was thus to assess which factors contribute to maternal death occurring during PPH. The factors were as follows: women's characteristics, aspects of pregnancy and delivery; components of PPH management; and organizational characteristics of the referral hospitals

\section{Discussion}

Buzaglo $\mathrm{N}$ did a retrospective cohort study and investigated risk factors for postpartum hemorrhage $(\mathrm{PPH})$ in vaginal deliveries and the influence of the previous PPH on the subsequent pregnancy. They saw that PPH complicated $0.8 \%$ of all first vaginal deliveries. Significant risk factors for $\mathrm{PPH}$ in vaginal delivery, were: post-term pregnancy, fertility treatments, hypertensive disorders, labor dystocia during the 2nd, and perineal tears grade 2 and 3, respectively. Previous PPH was found to be an independent risk factor for PPH in the subsequent pregnancy. Moreover, previous PPH was found to be a significant risk factor for cesarean section (CS) delivery, to complicate delivery with revision of uterus cavity, anemia, and to require a blood transfusion. They concluded that previous PPH poses a risk for recurrent PPH in subsequent delivery and an increased risk for CS. As PPH remains one of the major causes of maternal morbidity, this study strengthens the need for a comprehensive evaluation of prior PPH as a major risk factor for $\mathrm{PPH}$ recurrence. [1]. 
A prospective observational study was done by Briley $A$ et al on reporting errors, incidence and risk factors for postpartum haemorrhage (PPH) $(\geq 500$ $\mathrm{ml}$ ) and progression to severe PPH $(\geq 1500 \mathrm{ml})$. Sequential, interacting, traditional and new risk factors explain the highest rates of PPH and severe PPH reported to date.Gallos ID et al did a network meta-analysis on uterotonic agents for preventing postpartum haemorrhage. Objectives were to identify the most effective uterotonic agent(s) to prevent PPH with the least side effects, and generate a ranking according to their effectiveness and side-effect profile. The network meta-analysis included 196 trials (135,559 women) involving seven uterotonic agents and placebo or no treatment, conducted across 53 countries (including high-, middle- and low-income countries). The three highest-ranked uterotonic agents for prevention of $\mathrm{PPH} \geq 500 \mathrm{~mL}$ were ergometrine plus oxytocin combination, misoprostol plus oxytocin combination and carbetocin. There is evidence that ergometrine plus oxytocin (RR $0.70,95 \%$ CI 0.59 to 0.84 , moderate certainty), carbetocin (RR $0.72,95 \% \mathrm{CI}$ 0.56 to 0.93 , moderate certainty) and misoprostol plus oxytocin (RR $0.70,95 \%$ CI 0.58 to 0.86 , low certainty) may reduce $\mathrm{PPH} \geq 500 \mathrm{~mL}$ compared with oxytocin. [2,3].

High-certainty evidence suggests that misoprostol is less effective in preventing PPH $\geq 1000 \mathrm{~mL}$ when compared with oxytocin (RR 1.19, 95\% CI 1.01 to 1.42). Despite the comparable relative treatment effects between all uterotonics (except misoprostol) and oxytocin, ergometrine plus oxytocin, misoprostol plus oxytocin combinations and carbetocin were the highest-ranked agents for $\mathrm{PPH}$ $\geq 1000 \mathrm{~mL}$. The two combination regimens were associated with important side effects. When compared with oxytocin, misoprostol plus oxytocin combination increases the likelihood of vomiting (RR $2.11,95 \%$ CI 1.39 to 3.18 , high certainty) and fever (RR 3.14, 95\% CI 2.20 to 4.49 , moderate certainty). Ergometrine plus oxytocin increases the likelihood of vomiting (RR 2.93, 95\% CI 2.08 to 4.13 , moderate certainty) and may make little or no difference to the risk of hypertension, however absolute effects varied considerably and the certainty of the evidence was low for this outcome. All agents were generally effective for preventing $\mathrm{PPH}$ when compared with placebo or no treatment. Ergometrine plus oxytocin combination, carbetocin, and misoprostol plus oxytocin combination may have some additional desirable effects compared with the current standard oxytocin.
The two combination regimens, however, are associated with significant side effects.

Gilbert $L$ et al elaborated Postpartum haemorrhage continuing problem. The factors responsible for postpartum haemorrhage (PPH) in singleton vaginal deliveries, not complicated by a retained placenta, were identified by comparing labour characteristics in 86 women who had a PPH (blood loss $>500 \mathrm{ml}$ ) with 351 women whose blood loss at delivery was < $350 \mathrm{ml}$. Primiparity, induction of labour by amniotomy/ oxytocin, forceps delivery, long first and second stages, oxytocin com-pared with syntometrine (oxytocin plus ergometrine maleate), as a prophylactic oxytocic, were identified as significant risk factors. Epidural analgesia contributed indirectly to an increase in the risk of postpartum haemorrhage. The changes in labour ward practice over the last 20 years have resulted in the re-emergence of PPH as a significant problem. [4]. Sheiner E et al did a population-based study on obstetric risk factors and outcomes of pregnancies complicated with early postpartum hemorrhage. The study was aimed to identify obstetric risk factors for early postpartum hemorrhage (PPH) in singleton gestations and to evaluate pregnancy outcomes. Hypertensive disorder, failure to progress during the second stage of labor, oxytocin augmentation, vacuum extraction and LGA were found to be major risk factors for severe PPH. Special attention should be given after birth to hypertensive patients, and to patients who underwent induction of labour or instrumental delivery, as well as to those delivering LGA newborns. Similar study was done by Magann $E F$ et al who did an analysis of risk factors of postpartum hemorrhage after vaginal birth. $[5,6]$.

Joshi VM studied the role of internal iliac artery ligation (IIAL) in arresting and preventing postpartum haemorrhage (PPH). Out of 110 women who underwent IIAL, 88 had therapeutic IIAL for PPH from atony (36), genital tract injury (23), placenta praevia (21), placental abruption (4), uterine inversion (3) or coagulopathy (1). Hysterectomy was performed after IIAL failed to arrest haemorrhage in $33(39.3 \%)$ of 84 women (excluding 4 with vaginal lacerations). Hysterectomy was more likely with uterine rupture $(79 \%)$ than with nontraumatic PPH (up to $27 \%$ ). Failure to control haemorrhage by IIAL was evident immediately, and bleeding arrested by IIAL did not recur to require later laparotomy in any woman. It was concluded that IIAL is useful in the treatment and prevention of PPH from any cause. 
Early resort to IIAL effectively prevents hysterectomy in women with atonic $\mathrm{PPH}$. In traumatic PPH, IIAL facilitates hysterectomy or repair as indicated and prevents reactionary haemorrhage.[7]. McCormick ML reviewed the literature to determine the most effective methods for preventing postpartum hemorrhage $(\mathrm{PPH})$, the single most important cause of maternal death worldwide. A review of the literature confirms that active management of the third stage of labour, especially the administration of uterotonic drugs, reduces the risk of $\mathrm{PPH}$ due to uterine atony without increasing the incidence of the retained placenta or other serious complications. Oxytocin is the preferred uterotonic drug compared with syntometrine, but misoprostol also can be used to prevent haemorrhage in situations where parenteral medications are not available (e.g. at home births in developing countries). The use of active management of the third stage of labor to prevent $\mathrm{PPH}$ due to uterine atony should be expanded, especially in developing country settings. [8].

Similar were studies done by Tort $\mathrm{J}$ et al who did a cross-sectional epidemiological survey on factors associated with postpartum hemorrhage and maternal death. A better understanding of the factors associated with $\mathrm{PPH}$ maternal mortality is critical for preventing the risk of hospital-based maternal death. The purpose of this study was thus to assess which factors contribute to maternal death occurring during PPH. The factors were as follows: women's characteristics, aspects of pregnancy and delivery; components of $\mathrm{PPH}$ management; and organizational characteristics of the referral hospitals. They suggested that anemia should be diagnosed and treated before delivery and interhospital transfer of women should be improved, as well as the management of blood banks for quicker access to transfusion. Finally, an extent training of general practitioners in EmOC would contribute to the decrease of PPH maternal mortality. [9].

Grönvall $M$ et al did a retrospective case series on the use of Bakri balloon tamponade in the treatment of postpartum hemorrhage. Massive postpartum hemorrhage (PPH) is one of the most serious complications during delivery. Among the women treated with BBT, the cause of $\mathrm{PPH}$ was uterine atony $(16 \%)$, cervical rupture $(14 \%)$, vaginal rupture and/or paravaginal hematoma (22\%), placenta previa (18\%) and placental retention (30\%). The overall success rate was $86 \%$.
It was concluded that BBT is a simple, readily available, effective and safe procedure for the management of $\mathrm{PPH}$ in selective cases. BBT does not exclude the use of other procedures if necessary. Even if BBT failed, it may provide temporary tamponade and time to prepare for other interventions or transportation from a local hospital to a tertiary centre.

It was also suggested that BBT should be included in the PPH protocol.[10]. Managing postpartum hemorrhage depends in part on having a prepared mind, a complement of trained coworkers, and full access to modern therapies. The last 2 components are rare in resource-poor areas and their absence may be accentuated by climatic instability and lack of basic transportation. Karoshi $M$ et al emphasized greater use of the active management of the third stage of labor and administration of misoprostol by nontrained birth attendants that will provide beneficial reductions in hemorrhage rates in resource-poor areas.

Additional improvements depend on increasing public awareness, facilitating existing nongovernmental organizations in their communityrelated, upgrading training of traditional birth attendants, and providing cell phone communication to workers in remote areas, in addition to providing better access to blood.[11].

Another very important study was done by Marshall $A L$, Durani $U$ et al who studied the impact of postpartum haemorrhage on hospital length of stay and inpatient mortality as these outcomes have both clinical and economic significance. Over the study interval, postpartum hemorrhage occurred in $3 \%$ of deliveries. Among deliveries complicated by postpartum hemorrhage, $76.6 \%$ were attributed to uterine atony and $23.4 \%$ were nonatonic. They concluded that women with postpartum hemorrhage experienced significantly longer length of stay and higher inpatient mortality rates than women without postpartum hemorrhage, largely attributable to nonatonic causes of postpartum hemorrhage.

As hospital length of stay and inpatient mortality are important outcomes from both clinical and societal perspectives, interventions to reduce morbidity and mortality related to postpartum hemorrhage may simultaneously facilitate the delivery of more costeffective care and improve both maternal and population health. $[12,13]$. Abrams ET et al took an evolutionary and comparative perspective. 
Proximately, $\mathrm{PPH}$ results from the failure of the placenta to separate from the uterine wall properly, most often because of impairment of uterine muscle contraction.

It was argued that vulnerability to $\mathrm{PPH}$ stems from the intensely invasive nature of human placentation. The human placenta causes uterine vessels to undergo transformation to provide the developing fetus with a high plane of maternal resources; the degree of this transformation in humans is extensive. It was seen that the particularly invasive nature of the human placenta increases the possibility of increased blood loss at parturition. [14].

Kwast BE et al studied postpartum haemorrhage and its contribution to maternal mortality. Whereas Knight $M$ et al studied trends in postpartum hemorrhage in high resource countries. Our study was very much similar to the above studies and inference derived matched with the observations of the above authors. $[15,16]$.

\section{Conclusion}

Potential risk factors should be negated and new risk factors such as increased duration of labor, obesity and changes in second and third stage management practice should be revised. Training should be provided to all staff involved in maternity care concerning the assessment of blood loss and the monitoring of women after childbirth. This is key to reducing the severity of PPH and preventing any adverse outcomes.

\section{What this study adds to existing knowledge}

Clinicians should be more vigilant given the possibility that the frequency and severity of $\mathrm{PPH}$ have increased. This applies particularly to small hospitals with relatively few deliveries where management protocols may not be defined adequately and drugs or equipment may not be on hand to deal with unexpected severe PPH.

\section{Contribution by different authors}

First and Corresponding author: Dr. Neelima Agrawal : Data collection and statistical analysis. Second author Dr. P. N. Agrawal: Concept and data collection

\section{Reference}

01. Buzaglo N, Harlev A, Sergienko R, Sheiner E. Risk factors for early postpartum hemorrhage (PPH) in the first vaginal delivery, and obstetrical outcomes in subsequent pregnancy. J Matern Fetal Neonatal Med. 2015 May;28(8)932-7. doi: 10.3109/14767058.2014.937698

[PubMed][Google Scholar]

02. Briley A, Seed PT, Tydeman G, Ballard $H$, Waterstone $M$, Sandall J, et al. Reporting errors, incidence and risk factors for postpartum haemorrhage and progression to severe $\mathrm{PPH}-\mathrm{a}$ prospective observational study. BJOG. 2014 Jun;121(7)876-88. doi: 10.1111/1471-0528.12588 [Crossref][PubMed][Google Scholar]

03. Gallos ID, Papadopoulou A, Man R, Athanasopoulos $\mathrm{N}$, Tobias A, Price $M J$, et al. Uterotonic agents for preventing postpartum haemorrhage- a network meta-analysis. Cochrane Database Syst Rev. 2018 Dec 19;12(12)CD011689. doi: 10.1002/14651858.CD011689.pub3 [Crossref] [PubMed][Google Scholar]

04. Gilbert L, Porter W, Brown VA. Postpartum haemorrhage-a continuing problem. $\mathrm{Br} \mathrm{J}$ Obstet Gynaecol. 1987 Jan;94(1)67-71. doi: 10.1111/j.1471-0528.1987.tb02255.x [Crossref] [PubMed][Google Scholar]

05. Sheiner E, Sarid L, Levy A, Seidman DS, Hallak M. Obstetric risk factors and outcome of pregnancies complicated with early postpartum hemorrhage- a population-based study. J Matern Fetal Neonatal Med. 2005 Sep;18(3)149-54. doi: 10.1080/14767050500170088 [Crossref][PubMed] [Google Scholar]

06. Magann EF, Evans $S$, Hutchinson M, Collins R, Howard BC, Morrison JC. Postpartum hemorrhage after vaginal birth- an analysis of risk factors. South Med J. 2005 Apr;98(4)419-22. doi: 10.1097/01.SMJ.0000152760.34443.86 [Crossref] [PubMed][Google Scholar]

07. Joshi VM, Otiv SR, Majumder R, Nikam YA, Shrivastava $M$. Internal iliac artery ligation for arresting postpartum haemorrhage. BJOG. 2007 Mar;114(3)356-61. doi: 10.1111/j.14710528.2006.01235.x [Crossref][PubMed][Google Scholar] 
08. McCormick ML, Sanghvi HC, Kinzie B, McIntosh N. Preventing postpartum hemorrhage in lowresource settings. Int J Gynaecol Obstet. 2002 Jun;77(3)267-75. doi: 10.1016/s00207292(02)00020-6 [Crossref][PubMed][Google Scholar]

09. Tort J, Rozenberg $P$, Traoré $M$, Fournier $P$, Dumont A. Factors associated with postpartum hemorrhage maternal death in referral hospitals in Senegal and Mali- a cross-sectional epidemiological survey. BMC Pregnancy Childbirth. 2015 Sep 30;15;235. doi: 10.1186/s12884-015-0669-y [Crossref][PubMed][Google Scholar]

10. Grönvall M, Tikkanen M, Tallberg E, Paavonen J, Stefanovic V. Use of Bakri balloon tamponade in the treatment of postpartum hemorrhage- a series of 50 cases from a tertiary teaching hospital. Acta Obstet Gynecol Scand. 2013 Apr;92(4)433-8. doi: 10.1111/j.1600-0412.2012.01531.x [Crossref] [PubMed][Google Scholar]

11. Karoshi M, Keith L. Challenges in managing postpartum hemorrhage in resource-poor countries. Clin Obstet Gynecol. 2009 Jun;52(2)285-98. doi: 10.1097/GRF.0b013e3181a4f737 [PubMed][Google Scholar]

[Crossref]

12. Marshall $A L$, Durani $U$, Bartley $A$, Hagen $C E$, Ashrani A, Rose $C$, et al. The impact of postpartum hemorrhage on hospital length of stay and inpatient mortality- a National Inpatient Sample-based analysis. Am J Obstet Gynecol. 2017 Sep;217(3)344. e1-344.e6. doi: 10.1016/j.ajog.2017.05.004 [Crossref][PubMed] [Google Scholar]
13. Abrams ET, Rutherford JN. Framing postpartum hemorrhage as a consequence of human placental biology- an evolutionary and comparative perspective. Am Anthropol. 2011;113(3)417-30. doi: 10.1111/j.1548-1433.2011.01351.x [Crossref] [PubMed][Google Scholar]

14. Karoshi $M$, Keith $L$. Challenges in managing postpartum hemorrhage in resource-poor countries. Clin Obstet Gynecol. 2009 Jun;52(2)285-98. doi: 10.1097/GRF.0b013e3181a4f737 [PubMed][Google Scholar]

[Crossref]

15. Kwast BE. Postpartum haemorrhage- its contribution to maternal mortality. Midwifery. 1991 Jun;7(2)64-70. doi: 10.1016/s02666138(05)80229-3 [Crossref][PubMed][Google Scholar]

16. Knight $M$, Callaghan WM, Berg $C$, Alexander $S$, Bouvier-Colle $\mathrm{MH}$, Ford $\mathrm{JB}$, et al. Trends in postpartum hemorrhage in high resource countriesa review and recommendations from the International Postpartum Hemorrhage Collaborative Group. BMC Pregnancy Childbirth. 2009 Nov 27;9;55. doi: 10.1186/1471-2393-9-55 [Crossref] [PubMed][Google Scholar] 